\title{
Research on the Hill-start Control of Vehicles Based on the Pneumatic Auto Parking Brake System
}

\author{
Wang Hong-liang \\ Mechanical Engineer \\ Nanjing University of Science and Technology \\ Nanjing 210094, China \\ e-mail:whl343@163.com \\ Gao Fan-qin \\ Mechanical Engineer \\ Nanjing University of Science and Technology \\ Nanjing 210094, China \\ e-mail: 651549634@qq.com
}

\author{
Li Nan \\ Virtual Proving Ground \\ PLA General Armament Department \\ Nanjing 210028, China \\ e-mail: linan-24@163.com
}

\author{
Dai Xiao-ming \\ Mechanical Engineer \\ Nanjing University of Science and Technology \\ Nanjing 210094, China \\ e-mail: 1846040808@qq.com
}

\begin{abstract}
Focus on the hill-start auto control technology of the medium and heavy duty vehicles, structure of the spring brake cylinder and principle of the pneumatic auto parking brake system were analyzed. The force analysis of the vehicles which is on the hill was researched. The relationships between the driving force, the braking force and the grade resistance in the process of hill start were analyzed. The release time of the driving force was the key of hill-start control, and it influenced the process of hill start. The ideal control curve of the hill-start auto control was raised, and the release time of the parking brake was the time that the braking force could overcome the grade resistance. The control strategies of the pneumatic auto parking brake system were designed for the hill start, and the control software was written. The test vehicle was refitted, and the control strategy and software were written. The feasibility of the control strategy and software was proved on the test vehicle.
\end{abstract}

Keywords- vehicles; pneumatic; parking brake; hill-start; control strategy

\section{INTRODUCTION}

The hill-start control of vehicles is a difficult driving skill which need the driver coordination control clutch, accelerator and hand parking brake, relatively the error probability of manipulation is high ${ }^{[1,2]}$. Improper operation can cause the engine flameout and sliding, etc, leading to the road congestion, property damage or even Bodily Injury.

The auto parking brake control technology is a linear control technology which is based on the auto hand parking brake control technology ${ }^{[3,4]}$. It can change the traditional manual hand brake control into electronic button control by improving the structure of traditional hand brake like adding ECU, sensor and the execution component. Also it can implement auto parking brake and reduce the driver's labor intensity effectively.

The auto parking brake control technology can be applied to the process of hill-start ${ }^{[5]}$. Based on the force analysis of the vehicle which is on the hill, the essay put forward the idea which can come true the hill-start auto control by the automatic control of parking brake force and the coordination of clutch and engine. The structure and principle of the auto parking brake system of the medium and heavy duty vehicles were analyzed. The control method of the braking force was proposed. The control software was written and proved the strategy on the test vehicle, and the practicability was verified.

\section{AUto PARKING BRAKE SYSTEM OF THE MEDIUM AND HEAVY DUTY VEHICLES}

The medium and heavy duty vehicles need biggish parking brake force because of its weight. The pneumatic parking brake device is used due to the space of cab and limited output force from driver. The parking brake force comes from the strength spring in the rear axle brake cylinder, and it should be large enough to make parking brake come true. When the parking brake is released, the high pressure gas in the air tank compresses the strength spring, thus there is no stress on the hub. When the parking brake is applied, the high pressure gas is put out. So the parking brake system of the medium and heavy duty vehicles is also called "cut-off gas braking". This structure can ensure the long-term parking brake depending on the spring's elastic potential energy. The structure of spring brake cylinder in Fig . $1^{[6,7]}$. 


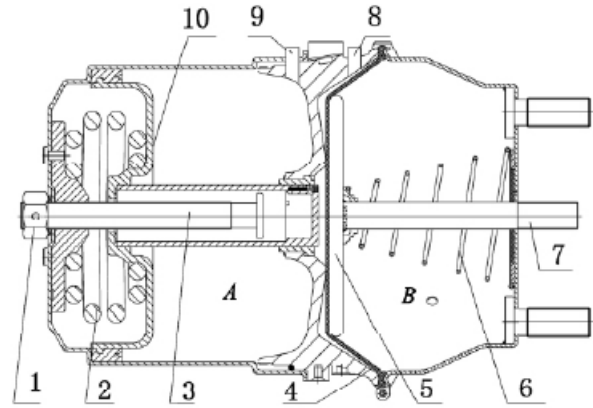

1-lock bolt 2- energy-storing spring 3-parking brake cylinder push rod 4-diaphragm 5-thrust plate 6-restoring spring 7-service brake cylinder push rod 8-service brake chamber air port 9-parking brake chamber air port 10-piston A-parking brake chamber B- service brake chamber

Figure 1. The structure of spring brake cylinder

The traditional parking brake system is controlled by the driver with manual valve. The auto parking brake system replaces the traditional manual valve by adding magnet valve which is in the gas path, and it achieves the automatic control by adding senor and ECU. The structure of the auto parking brake system in Fig . $2^{[8,9]}$.

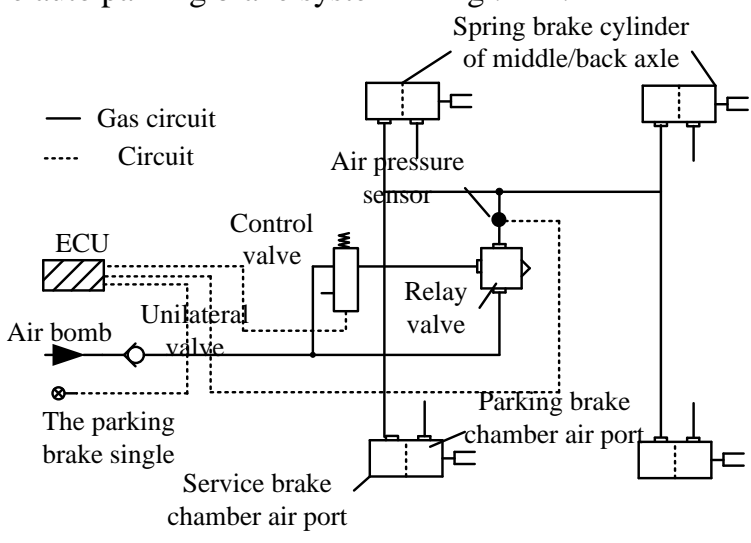

Figure 2. The pneumatic auto parking brake system

Through CAN bus, the ECU of the automatic parking brake system shares information with the vehicle electronic system and other electronic control system (automatic transmission, engine electronic control system, $\mathrm{ESP}, \mathrm{ABS}$, etc). Based on the vehicle operating condition, the ECU makes logical judgment of applying or releasing the parking brake, drives control valve, and finishes the process.

When the ECU makes the parking control valve to connect the gas path between the air bomb and the spring brake cylinder, high pressure gas from the air bomb is filled in the spring brake cylinder, and it compresses storage energy spring and the parking brake is released. When the parking control valve cuts off the gas path between the air bomb and the spring brake cylinder, high pressure gas is released into the atmosphere. The energy storage spring is reset. Then the parking brake is applied.

Moreover, the rake angle of vehicle is measured by rake angle sensor which is installed in the ECU, and the grade angle is also judged to make the automatic control of the system.

\section{THE FORCE ANALYSIS OF HILL-START}

When the vehicle starts on the road which has good adhesion condition, the deformation and wheel slip are ignored. The rolling resistance is smaller than the grade resistance, so the rolling resistance is ignored. Thus there is driving force, grade resistance, acceleration resistance and brake force on the vehicle which is on the hill, the force diagram in Fig .3.

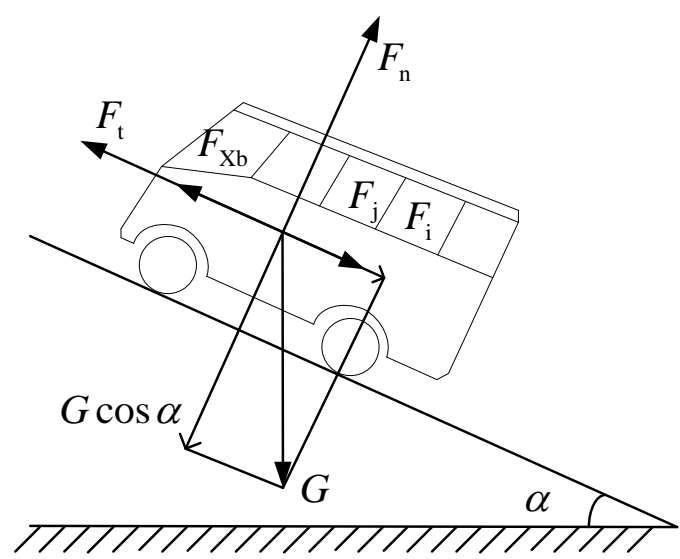

Figure 3. The vehicle which is on the hill force diagram

In Fig .3, $\quad F_{t}$ is driving force, $G$ is vehicle weight, $F_{n}$ is normal force, $F_{j}$ is acceleration resistance, $F_{X b}$ is brake force, $F_{i}$ is grade resistance, $\alpha$ is grade angle.

In the continuous progress of hill-start from static state to dynamic state, the direction and value of external force are constant changing especially the driving force, acceleration resistance and braking force interact on each other. Assuming that the driving force of vehicle is linearly increased, the force curve of hill-start can be described in Fig .4. The positive direction of each force is defined by direction shown in Fig .3.

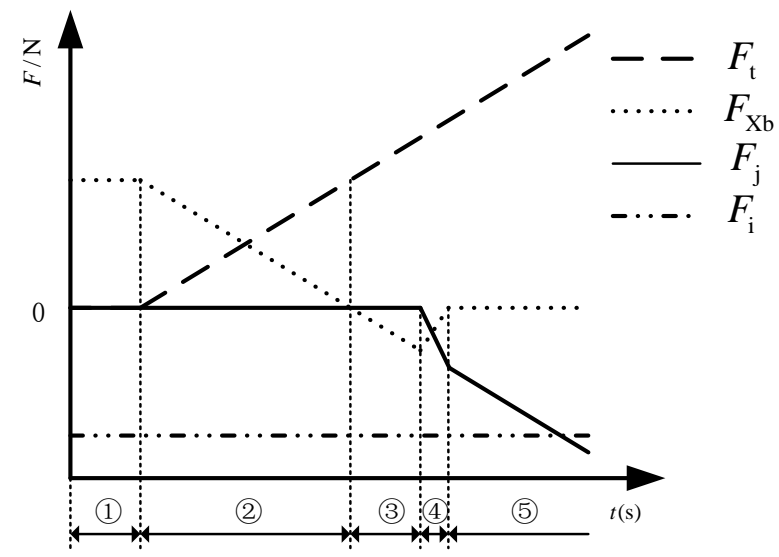

Figure 4. The force curve of hill-start

In Fig .4, based on the force changing curve, the hillstart is divided into five stages:

First stage: Vehicle is parking on the hill, and the speed is $0 \mathrm{~m} / \mathrm{s}$. The power which is from the engine is zero, so the driving force $F_{t}$ is zero. The braking force $F_{X b}$ and the grade resistance $F_{i}$ are balanced. They are in the opposite direction, and have the same size. 


$$
F_{\mathrm{Xb}}=F_{\mathrm{i}}
$$

Second stage: With the increase of driving force, braking force is decreased. When braking force is reduced to zero, the braking force $F_{X b}$ and the grade resistance $F_{i}$ are balance forces. They are in the opposite direction, and have the same size.

$$
F_{t}+F_{X b}=F_{i}
$$

Third stage: When the driving force $F_{X b}$ is larger than the grade resistance $F_{i}$, if the brake is not released in time. With the increase of driving force, the braking force is reverse increase and finally becomes the resistance force and it has the same added value with the driving force. Vehicle is still in the static state.

$$
F_{t}=F_{X b}+F_{i}
$$

Fourth stage: When the driving force increases to a certain value, the driver releases the parking brake. The packing force which is between brake shoe and hug is reduced. When braking force reversely increases to a specific value, the packing force has reduced to unable to output enough braking force. With the decrease of packing force, braking force is rapidly decreased until the brake is released, and the braking force is zero. When the braking force begins to be reduced, there exits acceleration resistance, acceleration and velocity, and the vehicle begin to run along the hill.

$$
F_{t}=F_{X b}+F_{i}+F_{j}
$$

Fifth stage: When the braking force is reduced to zero, the driving force is used to overcome the grade resistance and acceleration resistance. The vehicle velocity is increased.

$$
F_{t}=F_{i}+F_{j}
$$

The key of hill-start control is the third and fourth stage, and the key point of hill-start control as follows:

1. The brake is released until the driving force can overcome the grade resistance;

2. The brake is released in time, otherwise, the braking force is a hindrance in the process of hill start.

In the process of hill-start, the driving force is gradually increased while the grade resistance is stable. The key point of hill-start control is the time that the driving force equal to the grade resistance.

\section{StUdy On THE Auto Hill-START CONTROL STRATEGY}

To achieve auto hill-start control, the control system should have the following three functions:

1. Identifying the current grade resistance $F_{i}$;

2. Getting the current driving force $F_{t}$;
3. The automatic control of releasing the braking force;

The auto parking brake system has achieved the automatic control. It has the above three functions and has ability to realize auto hill-start control.

\section{A. Identifying the Grade Resistance}

The grade resistance depends on the vehicle weight and the current grade angle:

$$
F_{i}=G \sin \alpha=k \cdot G \sin \alpha_{1}
$$

$\alpha$ is the current grade angle, $\alpha_{1}$ is the body dump angle, $k$ is the safety factor.

The vehicle weight is acquired by the following ways:

1. Assembling the weight sensor for direct measurement;

2. Measuring the compression of the suspension system and the suspension stiffness to calculate the vehicle weight;

3 . The vehicle weight is calculated by traditional vehicle dynamics calculation formula ${ }^{[10]}$ and real-time information of the working condition.

After getting the vehicle weight, the grade resistance can be calculated by the formula (6), and it also can be used for the hill-start control.

\section{B. Getting the Driving Force}

Based on the mathematical model of the engine operating characteristics, the control unit of electric control engine can get the output torque by gathering the engine speed, fuel injection quantity and temperature. The calculated value is sent to the CAN bus. The ECU of auto parking brake system can get the engine output torque and the working state of the auxiliary system like air conditioner through CAN bus. Then the driving torque of the driving system and the driving force are both got. The formula is as follows:

$$
T_{t}=T_{e}-T_{a}
$$

$T_{t}$ is the driving torque of the driving system; $T_{e}$ is the current engine output torque; $T_{a}$ is the working torque of the auxiliary system.

\section{C. the Hill-start Control Strategy}

In the hill-start process, when the driving force can overcome the grade resistance, the parking brake is released. Therefore, when the auto parking brake system detects $T_{t}$ which is transmitted to the driving system by engine can overcome $F_{i}$, it gives order to the parking brake control valve, then the air tank and the spring brake cylinder are connected, and gas is filled in the spring brake cylinder. So the parking brake is released. The ideal hillstart control curve is shown in Fig .5. 


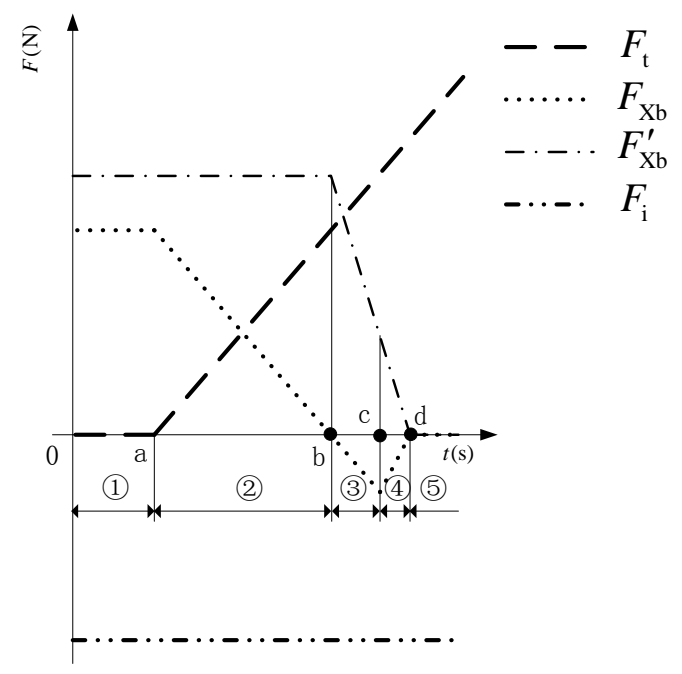

Figure 5. The ideal hill-start control curve

In Fig.5, point a is the moment that the driving force begins to increase; point $\mathrm{b}$ is the moment that the parking brake force decreases to zero with the increase of the driving force; point $\mathrm{c}$ is the moment of the best parking brake force; point $\mathrm{d}$ is the moment that the parking brake is completely released; $F_{\mathrm{Xb}}^{\prime}$ is the largest parking brake force.

$F_{X b}^{\prime}$ depends on the structure, size, the friction coefficient of the brake and the wheel radius, and it has an inverse relationship with the air pressure of the parking brake system. The calculation formula is as follows:

$$
F_{X b}^{\prime}=\frac{T_{\mu}}{r}
$$

$T_{\mu}$ is the friction torque which could appear in a situation that brake friction plate and drum(or plate) have relative slip $(\mathrm{Nm})$.

When vehicle is in the static state, the parking brake force belongs to the static friction force, and its value depends on the grade resistance and the driving force. Meanwhile, its maximum of the parking brake force is limited to the brake pressure which is between the brake friction plate and drum. The formula is as follows:

$$
F_{X b}=\left\{\begin{array}{l}
F_{i}-F_{t} \\
\leq F_{X b}^{\prime}
\end{array}\right.
$$

The hill-start is divided into five stages:

First stage: When the vehicle is on the hill, the maximum braking force is achieved by the spring brake cylinder. The braking force can overcome the grade resistance and can ensure the reliable parking brake when the vehicle is on the hill. The clutch is not combined, and no driving torque is transmitted to the wheels. At this time, the parking brake force is the static friction force, and its value equals to the grade resistance. The formula is as follows:

$$
F_{X b}=F_{i}
$$

Second stage: This is the initial phase of the hill-start. The driver hangs up the start gear and hits the accelerator, and the clutch is slowly combined. The driving torque which is transmitted to wheel is gradually increased until it can overcome the grade resistance. With the increase of driving force $F_{t}$, the parking brake force $F_{X b}$ is gradually decreased to zero. The formula is as follows:

$$
F_{X b}=F_{i}-F_{t}
$$

Third stage: When the driving torque can overcome the grade resistance, the automatic parking brake system gives order to the parking brake control valve. Then the air tank and the spring brake cylinder are connected, and gas is filled in the spring brake cylinder. So the parking brake is released. It takes definite time cycle to release the parking brake. Before the parking brake is not completely released, the driving torque is increased. The parking brake force is reversely increased, and it impedes the vehicle movement. The formula is as follows:

$$
F_{X b}=F_{t}-F_{i}
$$

Fourth stage: With the release of the parking brake, the brake pressure which is provided by the spring brake cylinder cannot support the increase of the parking brake force. The largest parking brake force $F_{X b}^{\prime}$ has been less than the difference between the driving force and the grade resistance. With the further release of brake, the parking brake force is reduced to zero. The formula is as follows:

$$
F_{X b}=F_{X b}^{\prime}=\frac{T_{\mu}}{r}
$$

Fifth stage: The parking brake system has been completely released. Then the vehicle can drive on the hill well.

In the process of hill-start, point $\mathrm{b}$ is the key to success. The correlation between the parking brake releasing moment and point $b$ is as follows:

1. The parking brake is released until the driving force overcomes the grade resistance. Otherwise, slide will easily happen;

2. When the driving force overcomes the grade resistance, the parking brake is released as soon as possible. Otherwise, it will cause unnecessary wear between the braking system and the transmission system, and the start control effect is reduced.

To achieve the best effect, the release moment of the parking brake which is in the process of the hill-start is point $b$. The control software is written to implement it.

\section{CONTROL SOFTWARE}

According to the above control strategy, the control software is written. The process is shown in Fig .6. 


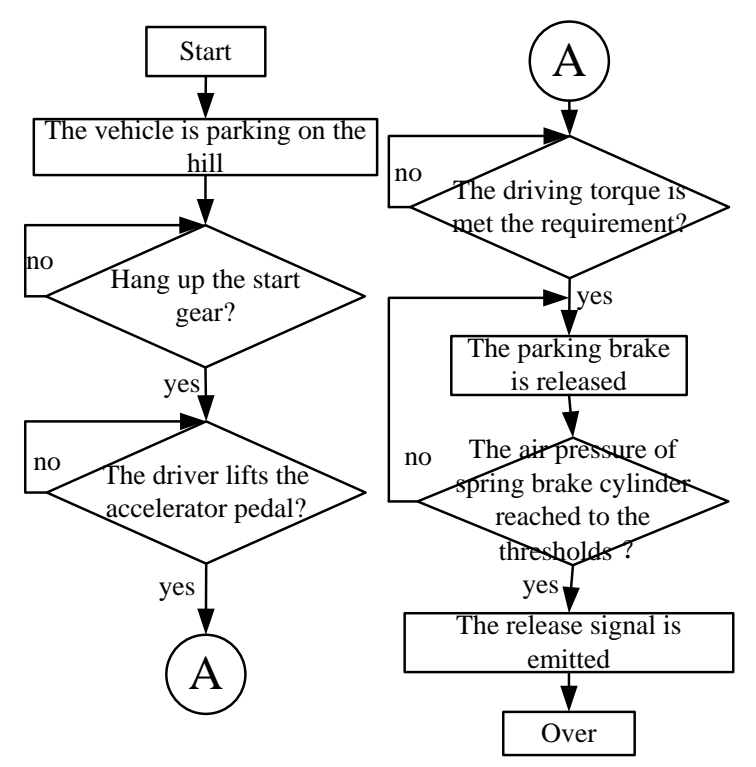

Figure 6. The process of control software

In the process of hill start, firstly, the vehicle has been reliably parked on the ramp. Moreover, the system judges driver's intention. After the driver hangs up the start gear and lifts the brake pedal, the vehicle has been in the process of hill start. According to the angle sensor signals and the weight information, the auto parking brake system calculates the grade resistance. At the same time, the driving torque of the engine is detected. Finally, when the driving force can overcome the grade resistance, the parking brake is released by the control valve which is driven by the auto parking brake system. The release signal is emitted. Then the vehicle drives on the hill well.

\section{EXPERIMENT}

The auto parking brake system is installed on a medium passenger car. The vehicle is refitted. The test vehicle is shown in Fig .7.

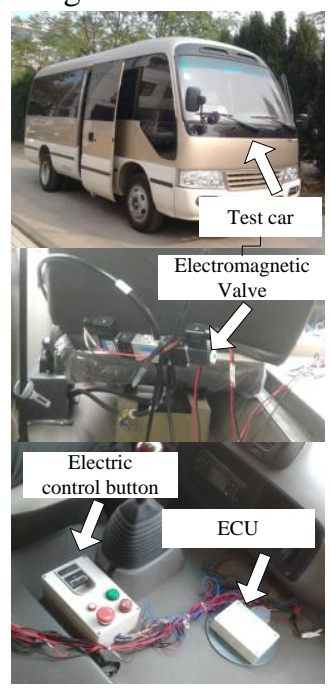

Figure 7. Test vehicle and its auto parking brake system

The control strategy and software of hill start were proved on the test vehicle, based on the auto parking brake system. The experimental data is shown in Fig .8.
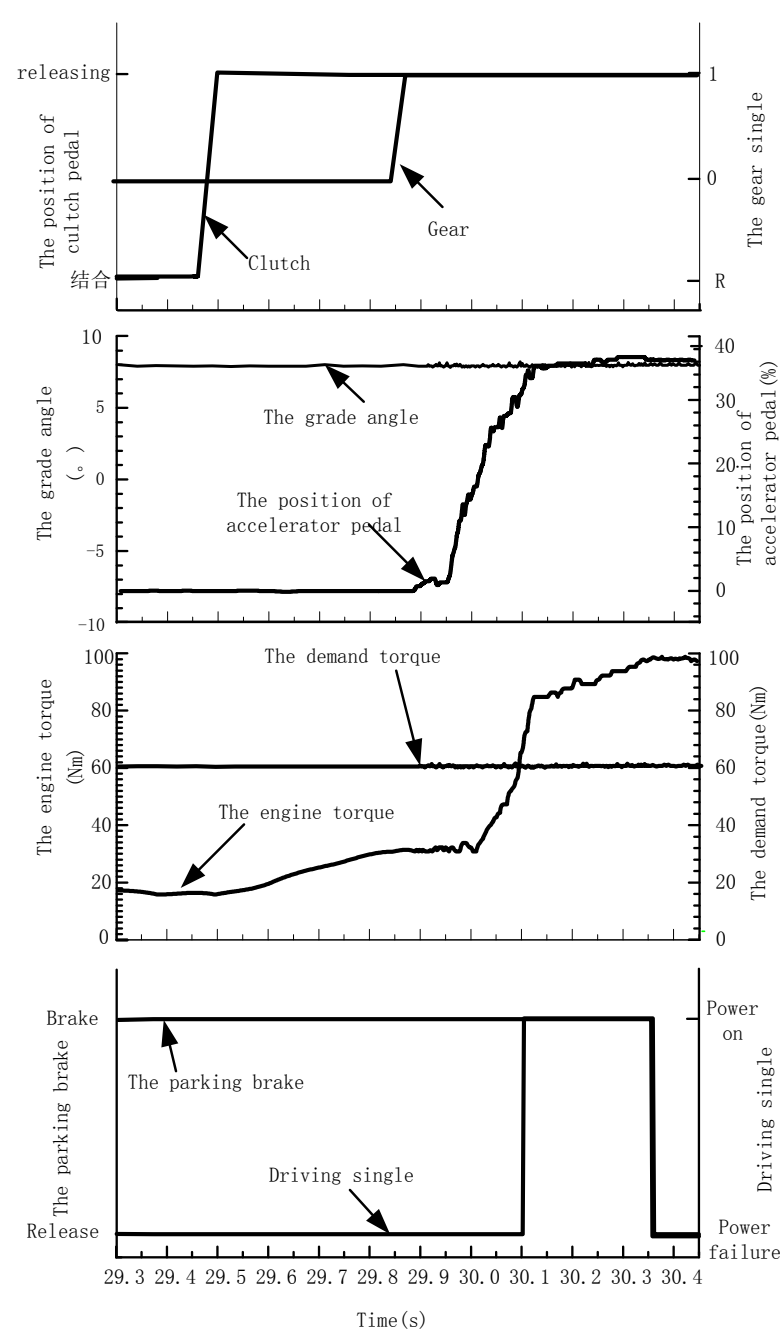

Figure 8. Hill-start experimental data based on the auto parking brake system

In fig .8 , when the vehicle started on the hill which grade angle is $8^{\circ}(14 \%)$, the vehicle was parked on the hill. The driver pushed the clutch pedal down at $29.45 \mathrm{~s}$ and hung up the first gear at 29.85s. When the driver lifted the clutch pedal, oil was smoothly gave. Clutch delivered the driving torque which was gradually increased. After the engine driving torque was larger than the torque which was needed by the vehicle hill-start (30.1 s), the control valve was started. And the gas was filled in the spring brake cylinder. At $30.35 \mathrm{~s}$, the spring brake cylinder air pressure reached to the thresholds. The switch signal of air pressure was changed. The parking brake had been released. Then the vehicle drove on the hill well.

\section{CONCLUSION}

Through the work of this paper, the control strategy and software of the medium and heavy duty vehicles have been implemented for hill start, based on the automatic parking brake system. Structure and principle of the pneumatic auto parking brake system were analyzed. Based on the force analysis of the vehicles which is on the hill, the requirements of hill-start auto control were raised. The control strategy of the pneumatic auto parking brake system was designed for the hill start. The control software 
was written. The feasibility of the control strategy and software was proved on the test vehicle.

\section{ACKNOWLEDGMENT}

R.B.G thanks the National Natural Science Foundation of China No. 51205204, the National Natural Science Foundation of China No. 51205209, China Postdoctoral Science Foundation No. 2013M531360.

\section{REFERENCES}

[1] Ge An-lin, Lei Yu-long, and Gao Yi-feng, "A Research on Hill Starting Control of AMT System of Vehicle," Automotive Engineering, vol. 20, no. 3, pp. 150-164, 1998.

[2] Olivier Cayol, Alessandro De Rinaldis, and Christophe Desfriches, "Method for Assisting with Hill Starts", U.S. Patent 20110202245A1, Aug. 18, 2011.

[3] Minseok Jang, Young O.Lee, and Wongoo Lee, "Novel Clamping Force Control for Electronic Parking Brake System," in
Proceedings of the 7th Asian Control Conference, 2009, pp. 15881593.

[4] Harald Klode and Larry R.Miller, "Electromechanical Parking Brake", U.S. Patent 6561321B1, May.13, 2003.

[5] Alessandro Monti and Richard Pothin, "System and Method foe Controlling the Release of an Automatic Parking Brake Device Onboard an Automobile", U.S. Patent 20100262329A1, Oct. 14, 2010.

[6] Lain Fantazi and Franck Hemery, "Spring brake accumulator cylinder”, U.S. Patent 7866765B2, Jan. 11, 2011

[7] Wang Hongliang, Zhao Xijun, and Liu Haiou, "The electronicpneumatic hill-starting assist control foe heavy-duty vehicles with AMT, " Journal of Shandong University (Engineering Science), vol. 39 , no. 5 , pp. $79-83,2009$.

[8] Wang Xianhui, "The pneumatic intelligent electronic parking brake system", China. Patent 203032658U, Jul. 03, 2013.

[9] Wang Hongliang, "The pneumatic adjustable electronic parking brake system”, China. Patent 203391766U, Jan. 15, 2014.

[10] Jin Hui, Ge Anlin, and Qin Guihe, "Study on Slope Recognition Method based on Vehicle's Longitudinal Dynamic," Chinese Journal of Mechanical Engineering, vol. 38, no. 1, pp. 79-86, 2002. 